\title{
Diseño de Prácticas de Laboratorio en Electrónica con TICs
}

\author{
Jesús E. Cano \\ Departamento de Electrónica, Facultad de Tecnología y Ciencias Aplicadas, Universidad \\ Nacional de Catamarca, Argentina \\ jesuseduardocano@gmail.com
}

\section{María V. Poliche}

Departamento de Electrónica, Facultad de Tecnología y Ciencias Aplicadas, Universidad Nacional de Catamarca, Argentina

vpoliche@hotmail.com

\section{Paola I. Beltramini}

Departamento de Electrónica, Facultad de Tecnología y Ciencias Aplicadas, Universidad Nacional de Catamarca, Argentina

palobel2012@gmail.com

\section{Sergio H. Gallina}

Departamento de Electrónica, Facultad de Tecnología y Ciencias Aplicadas, Universidad Nacional de Catamarca, Argentina

shgallina@hotmail.com

Presentación 18/11/2016

Aprobación 20/07/2017

\section{Resumen}

En este trabajo se presenta una propuesta de estrategia metodológica basada en el aprendizaje experiencial de Kolb, para ser aplicado a la enseñanza de la Electrónica Analógica mediante la implementación de laboratorios portátiles de escritorio en diferentes asignaturas de la carrera Ingeniería en Electrónica (UNCa). El proceso constara de dos etapas, la primera de ellas principalmente técnica con desarrollo de un prototipo de laboratorio portátil de escritorio y la segunda, mediante la investigación-acción, llegar a implementar su aplicación a la enseñanza. Para la primera etapa se abordaran las siguientes fases de desarrollo: la electrónica, el software asociado, las comunicaciones y los desarrollos de las clases. En la segunda etapa una vez desarrollado el prototipo se pretenderá introducir en la enseñanza de cátedras de las carreras de ingeniera y tecnicatura de la Facultad de Tecnología y Ciencias Aplicadas de la UNCA

Palabras clave: Aprendizaje experiencial, TICs, Laboratorios, Electrónica 


\begin{abstract}
This paper presents a proposal of a methodological strategy based on Kolb's experiential learning to be applied to the teaching of Analog Electronics through the implementation of portable desktop laboratories in different degree of the Electronic Engineering (UNCA). The process consists of two phases, the first of them mainly technical with development of a portable laboratory prototype and the second, through research, implement its application in education. For the first phase the following steps of development are: electronics, software, communications and classroom developments. In the second phase, once the prototype has been developed, it will be attempted to introduce in education of the courses of engineering and technicature of the Faculty of Technology and Applied Sciences (UNCA).
\end{abstract}

Keywords: Experiential learning, TICs, laboratories, Electronics

\title{
Introducción
}

Las clases en las carreras de ingeniería electrónica se han llevado a cabo a través del desarrollo de clases magistrales, exposiciones de estudiantes, prácticas de laboratorio y proyectos prácticos, cuyo ámbito en la mayoría de los casos no ha ido más allá de los contenidos del curso. Adicionalmente algunos alumnos se incorporan a proyectos de investigación donde complementan su formación.

Muchos docentes de carreras tecnológicas, carentes de preparación psicopedagógica y en didáctica docente, perciben la necesidad de innovar en el aula, incluyendo además de la lógica actualización de contenidos, conocimientos sobre teorías de aprendizaje, metodologías de enseñanza y técnicas didácticas para lograr la optimización del proceso de enseñanza - aprendizaje y poder afrontar el reto que representan las nuevas generaciones de estudiantes.

Esta doble necesidad de cambio, la actualización y el indisimulable rasgo distintivo de las nuevas generaciones de estudiantes, plantean la imperiosa necesidad de encontrar una metodología y estrategias de enseñanza-aprendizaje eficaz para el área de la electrónica, pilar básico para las carreras de grado en las que los estudiantes deben ver reflejados al máximo su vocación por la carrera y comenzar a realizar actividades similares a las propias de su profesión, ya que son asignaturas de indudable contenido práctico.

Es por eso que surgen en esta instancia varios interrogantes: ¿los alumnos de esta generación aprenden igual que los de generaciones anteriores?, ¿cuál es el método de enseñanza más apropiado para la carrera de Ingeniería Electrónica?, ¿todos los métodos son válidos para cualquier alumno?, ¿cómo lograr llegar a todos los alumnos?, ¿por qué se olvida lo aprendido?. Siendo consciente de que no existe una única forma de enseñar, se propone en este trabajo una forma de enseñar constructivista basada en lo que se denominará Laboratorio de Escritorio.

La propuesta presenta como objetivo central trabajar en la generación de competencias y habilidades de desempeño en el alumno de ingeniería desde una etapa temprana de su formación académica. La incorporación de laboratorios usando las nuevas TICs (tecnologías de la información y la comunicación) pretende incentivar 
la capacidad de resolución de problemas cercanos a la vida profesional integrando el saber, saber hacer y saber ser, a través de la promoción de la automotivación, la toma de decisiones, la comprensión, el análisis y la síntesis, el uso de estrategias adecuadas en la búsqueda de la solución más pertinente y el trabajo grupal colaborativo.

\section{Marco teórico}

La inclusión de tecnología, dentro y fuera del aula, permite incorporar otros mecanismos de enseñanza y aprendizaje para complementar o potenciar la enseñanza formal tradicional.

La enseñanza de la electrónica y, en particular, la aproximación a los conocimientos básicos de la misma, debe producirse a través de las acciones provocadas por una experiencia concreta, de manera de transformarse en una conceptualización abstracta que le permite aplicarla a nuevas situaciones, formando un proceso continuo e interactivo que genera nuevos aprendizajes.

Dentro de múltiples enfoques que se pueden tomar como base de estas ideas está el denominado "aprendizaje experiencial".

Uno de los referentes de esta corriente fue John Dewey filósofo, pedagogo y psicólogo estadounidense que manifestó que la construcción de conocimiento a partir de una experiencia concreta se representa como un proceso cíclico de interrelación entre cuatro fases: la experiencia concreta, la reflexión, la conceptualización y la aplicación (González, Mónica L et. al, ). De esta manera el aprendizaje es un proceso de relación mutua entre experiencia y teoría, no basta con una experiencia para producir conocimiento, es necesaria la modificación de las estrategias cognitivas del sujeto.

La experiencia cobra sentido cuando se vincula con el conocimiento previo y se desarrollan andamiajes conceptuales que permitan aplicar el nuevo conocimiento a nuevas situaciones (González, Mónica L et. al,). En este proceso el estudiante formula hipótesis, experimenta y aplica su creatividad para llegar a la solución desarrollando conocimiento.

Estos modelos de aprendizaje se contraponen con el modelo tradicional de aprendizaje que promueve un individuo pasivo, receptor de información que transformará en conocimiento a su debido tiempo. La propuesta de aprendizaje experiencial se adapta a las nuevas demandas profesionales vinculadas con la formación y desarrollo de competencias y habilidades.

Se pueden mencionar diversas estrategias basadas en el aprendizaje significativo constructivista en el marco del denominado aprendizaje experiencial, tales como (González, Mónica L et. al, ):

- Solución de problemas reales (aplicación a situaciones de un ámbito de conocimiento donde la solución puede no ser única)

- Análisis de casos (promueven habilidades de aplicación e integración de conocimiento, toma de decisiones y solución de problemas)

- Aprendizaje basado en proyectos (el alumno aprende a través de una experiencia personal y activa orientada a la solución de problemas específicos) 
- Trabajo en grupo

- Simulaciones de contextos reales (laboratorios virtuales)

- Aprendizajes mediados por TICs.

Otro referente de esta corriente de aprendizaje fue David Kolb, un experto en administración de la Universidad Case Western Reserve, quien desarrolló un modelo de aprendizaje en 1981 como un proceso que permite deducir conceptos y principios a partir de la experiencia para orientar la conducta a situaciones nuevas, es decir, "un proceso mediante el cual se crea conocimiento a través de la transformación de la experiencia" [Kolb D., 1974].

El modelo de aprendizaje queda constituido por cuatro etapas que se relacionan (Kolb D., 1981):

- experiencia concreta

- observación reflexiva

- conceptualización abstracta

- experimentación activa

El núcleo del modelo es una sencilla descripción del modelo de aprendizaje, de cómo se traduce la experiencia en conceptos que se emplean a su vez como guías de elección de nuevas experiencias.

Basándose en la aplicación de este modelo se propondrán actividades curricu-lares con el objetivo de proponer un aprendizaje motivador y significativo usando herramientas provistas por las tecnologías.

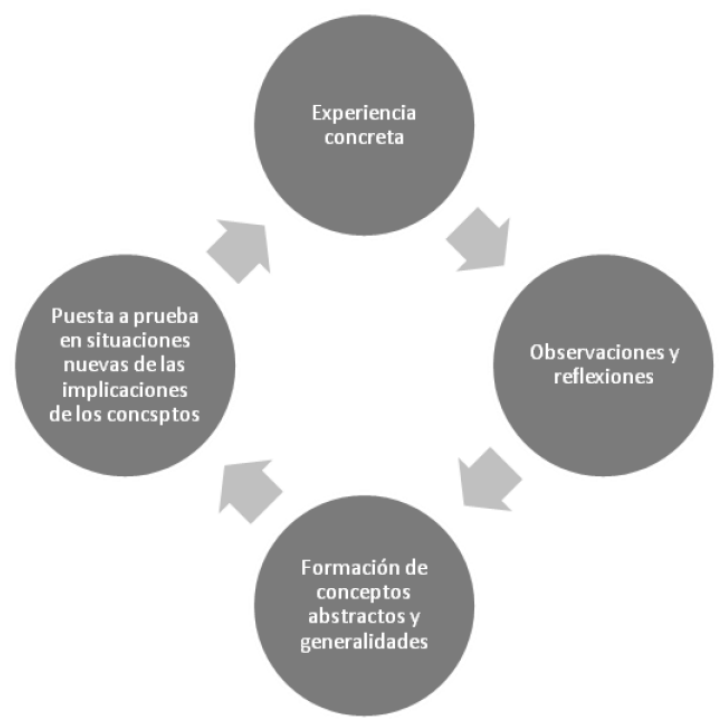

Fig. 1. El modelo de aprendizaje mediante experiencia. Fuente: Kolb D., "Learning styles and disciplinary differences",

Esta es una perspectiva diferente que requiere de un posicionamiento no tradicional de la práctica docente. 
El desarrollo alcanzado por las actuales tecnologías de información y comunicación (TICs) posibilitan hoy, en el área de las enseñanzas científicas y tecnológicas, nuevos modos de acercamiento al conocimiento basados, entre otros, en el desarrollo de laboratorios virtuales y remotos.

\section{Viabilidad educativa de los laboratorios utilizando TICs}

El sistema tradicional de la enseñanza en los laboratorios se distinguen con frecuencia por el carácter no auténtico del discurso pedagógico, ya que a veces las preguntas que formula un profesor no son tales (en tanto sólo las plantea porque conoce las respuestas), y en que los problemas por resolver que plantea pueden considerarse como "problemas de juguete". Esta última analogía se refiere al perfil de los problemas, que si bien en apariencia guardan relación de semejanza con problemas reales, no presentan ni la complejidad ni el juego de variables o determinaciones que pertenecen al mundo de la vida real. La utilización de TICs, como ser programas de simulación, o mejor aún, de software y hardware, permiten generar ambientes de aprendizaje basados en experimentos de laboratorio y resolución de problemas de ingeniería reales, fortaleciendo el desarrollo de competencias y desempeños necesarios para la práctica profesional del futuro ingeniero.

Actualmente en la carrera de Ingeniería Electrónica, las prácticas son realizadas en un entorno de laboratorio real y constituyen actividades que permiten a los alumnos consolidar conocimientos a través de la experimentación con la realidad, la manipulación de equipos y datos, instrumentos y circuitos eléctricos. Estas actividades presentan varios problemas los que han sido y son constantemente analizados por los docentes y además han sido objeto de una investigación emprendida por el Departamento Académico de la carrera de Ingeniería Electrónica. Los resultados de esta investigación arrojaron las siguientes conclusiones:

- Los estudiantes en una amplia mayoría (más del $80 \%$ ) piensan que las prácticas de laboratorio siempre son necesarias, que van con mucha motivación a ese tipo de clases, que hace falta una explicación teórica previa siempre y que debería haber más horas de clase de laboratorio.

- Más de un $60 \%$ cree conveniente experimentar una renovación en las clases introduciendo nuevas metodologías y didácticas.

- En cuanto a la calidad de las clases, las opiniones se dividen: un 50\% opina las consignas enunciadas no están bien detalladas y además que hay problemas de desfasaje en el tiempo, entre la clase de teorías de la clase de práctica.

- Respecto a la metodología para hacer la experiencia un 37,5\% prefiere hacerla en forma individual, en igual porcentaje prefieren trabajar sólo en grupo de a dos alumnos y un $25 \%$ restante creen que la modalidad depende del ensayo que se trate. Con respecto a grupos de 3 o más no son del gusto del $100 \%$ de los entrevistados e incluso adicionalmente se quejan de que 
siempre hay integrantes que no aportan y por lo tanto desnaturalizan el objetivo del trabajo grupal.

- Las quejas sobre el estado de los instrumentos es de un $50 \%$ que ve demoras cuando por falta de mantenimiento hay equipos fuera de servicio o incluso producen errores en los ensayos con pérdidas de tiempo.

- Sobre las metodologías usadas por los docentes se sienten muy conformes con el desafío permanente de trabajar y manipular los componentes ya que los enfrenta a problemas reales, pero les disgusta tener que hacer los cálculos de circuitos, que le consumen un tiempo valioso para hacer experiencia con los recursos del laboratorio y así les resulta escaso el tiempo para completar un objetivo dado.

En virtud de mejorar estas variables analizadas y basándose en las consignas del modelo de aprendizaje experiencial se propone el uso de un laboratorio de escritorio portátil, pretendiendo con este recurso obtener las ventajas de los laboratorios virtuales y de los laboratorios remotos.

Un "laboratorio virtual" es un sistema basado en el uso de la computadora y software específico de aplicación que aproxima el ambiente de trabajo al de un laboratorio tradicional. Se realizan experiencias similares utilizando aplicaciones informáticas que incluyen el armado esquemático del circuito, manipulación de instrumentos, visualización de animaciones, obtención numérica y gráfica de resultados, incluyendo en algunos casos el almacenado digital para procesamiento posterior con programas de graficación más complejos y bases de datos.

En un "laboratorio remoto" se opera contra un proceso físico real ya que tiene equipos físicos que realizan los ensayos localmente, pero en los que el usuario accede en forma remota a través de una interface que está implementada mediante software que permite hablar de un trabajo experimental con un desarrollo de verdaderas prácticas de laboratorio en tanto el alumno, a través de la red, de manera interactiva, opera con dispositivos reales. También se logra experimentación simultánea de grupos de alumnos con un mismo equipamiento, posibilitando compartir recursos entre instituciones en el marco de dictados de asignaturas con propósitos de "e-learning".

Por su parte, un "laboratorio de escritorio portátil" aquí propuesto no presenta una realidad simulada, sino como el remoto presenta un sistema real, pero tampoco pretende que sólo el software interactúe con el alumno, lo guíe haciendo que el progreso de la enseñanza depende en gran medida del dominio del software. En el laboratorio de escritorio portátil el alumno realiza las prácticas según su construcción teórica, aplica los conocimientos adquiridos y los relaciona en las diversas propuestas de diseño. En los otros ejemplos de laboratorio no se manipulan los componentes, solo se manejan imágenes de archivo o se eligen a través de la red de comunicación.

Los laboratorios de escritorio presentan mayores ventajas debido a que éstos proporcionan un mayor nivel de interactividad y el alumno entra en contacto con equipamiento real, en lugar de entrar en contacto con programas simulados.

En resumen estos laboratorios con TICs no tradicionales poseen dos características distintivas. La primera sería la interacción que presupone trabajar 
sobre un sistema informático, el alumno solo progresa si se suministran ciertas respuestas, las que son validadas por el sistema y el mismo sistema puede proporcionar ayudas o tutorías, más aun si el laboratorio está en una red educativa con el profesor y el curso. La segunda característica es la simulación, aquí el alumno no manipula los componentes, no tiene fallas constructivas (soldadura fría por dar un ejemplo en electrónica). Lo que se hace en el laboratorio virtual no es real y lo que se hace depende mucho de cuanto se ha leído el manual de uso. Lo que se hace en un laboratorio remoto no permite la manipulación de los componentes ni entrar en fallas que si se dan en la vida real.

El objetivo fundamental de los trabajos de laboratorio es fomentar una enseñanza activa y participativa, donde se impulse el espíritu crítico. De este modo se favorece que el alumno: desarrolle habilidades, aprenda técnicas elementales y se familiarice con el manejo de componentes, instrumentos y aparatos. Para que esto funcione adecuadamente, es fundamental plantear correctamente las experiencias.

Las prácticas en estos laboratorios basados en TICs deben regirse por ciertos aspectos, muy similares a las prácticas en un laboratorio real, entre los cuales podemos mencionar:

- Visión: Las prácticas deben ofrecer una visión de la electrónica en todas sus áreas de trabajo. Se fomentará la identificación y solución de problemas con una base matemático-teórica.

- Multidisciplinar: control electrónico de procesos, tratamiento de señales, comunicaciones digitales, etc. De esta manera se buscará la integración de conocimientos.

- Realismo: Las prácticas deben ser realistas en la medida de lo posible y deben abordar el diseño de un sistema completo desde el punto de vista de su funcionalidad, y real desde el punto de vista de su posible implementación.

- Perspectiva: Los laboratorios tendrán un propósito fundamentalmente docente y mostrarán al alumno las diversas alternativas de diseño posibles, intentándose mostrar el contraste entre las posibles soluciones posibles a un problema.

- Enunciado: La especificación del problema básico se realizará por medio de un enunciado de una longitud y nivel de detalle apropiados.

- Iniciativa: Se fomentará la iniciativa del alumno.

- Evaluación: La evaluación considerará tanto aspectos de funcionamiento como de dominio del diseño, el montaje, las pruebas y la documentación del prototipo desarrollado.

\section{DESARROLLO}

Características del laboratorio portátil de escritorio propuesto para la enseñanza de electrónica

Para efectos del diseño se abordan tres aspectos de desarrollo: el hardware o electrónica, el software y la metodología a aplicar para la enseñanza aplicando 
esta nueva herramienta. A continuación se describen cada uno de ellos según el siguiente detalle:

\section{Desarrollo electrónico}

Será el encargado de proveer el hardware del "laboratorio", permitirá la generación de señales eléctricas y su control. Es decir, la electrónica provee los equipos o dispositivos con los cuales se ingresa, extrae, presenta o procesan datos, información o servicios.

El laboratorio propuesto se centra en la Computadora Industrial Abierta Argentina (CIAA) y consta de siete bloques diferentes que le brindan potencialidad al laboratorio: Fuente de alimentación, Display gráfico y táctil, Instrumentos, Generador de señales, el Módulo de conectividad que brinda comunicación a redes de datos; adicionalmente consta de un conector donde se tienen disponibles las diferentes señales para ensamblar las placas de circuitos intercambiables para cada tipo de clase a diseñar.

El siguiente esquema funcional ilustra la relación de cada uno de estos bloques:

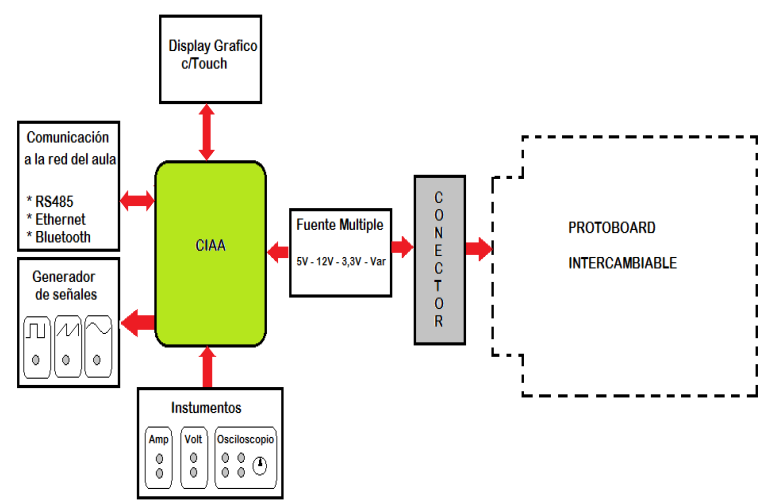

Fig. 2. Diagrama en bloques del desarrollo propuesto. Fuente: elaboración propia.

\section{Desarrollo de software}

Se distingue aquí diferentes niveles de software según la función o misión que deberá cumplirse. De esta manera el software se organizará de manera que cada paquete se encargue de funciones bien específicas, a saber: una rutina que lleve a cabo la lectura de los datos directamente de la placas electrónica diseñadas para cada clase, el procesamiento y conversión de la información a valores digitales adecuados para su correcto tratamiento, una rutina que atienda las solicitudes que los usuarios (estudiantes) hagan por la pantalla táctil y permita además una interfaz donde se pueda exponerles claramente las situaciones de configuración haciendo lo más autónomo posible el desarrollo de cada práctico con las particularidades de cada una de las experiencias que se les proponga.

También se debe prever una rutina de programación que permita "subir" todo el proceso de la clase hacia una interfaz de comunicación para que desde una computadora se permita interactuar a docentes con alumnos en forma amigable, simple y eficaz. 


\section{Desarrollo de las clases}

Considerando necesario acotar este punto por cuanto la variedad de clases es muy amplia, y teniendo en cuenta las asignatura de la carrera, se cree provechoso realizar el diseño de las placas para el desarrollo de clases que tengan que ver con los siguientes tópicos: polarización de transistores y diodos, control de motores de corriente continua y control de posicionamiento de precisión de un carro que se desplaza sobre guías rectas. Cada clase o tema incluye el diseño del aprendizaje teórico, de las prácticas a realizar por el alumno y el mecanismo de evaluación.

Como este es el punto más importante a definir en este artículo se presenta seguidamente el diseño de una clase sobre el conocimiento de los transistores bipolares.

\section{Diseño de las clases usando el laboratorio portátil de escritorio}

teniendo en cuenta que este proyecto se encuentra en su fase de iniciación por ahora se presentan las pautas generales de diseño para hacer de esta clase un aprendizaje experiencial. Y para ser más acotado aún, el esquema de la clase será para un tema seleccionado de la Electrónica: "Estudio del transistor bipolar".

Los objetivos de los ensayos son los siguientes:

- Familiarizarse con los transistores, la identificación de sus terminales y la forma como se emplean en circuitos prácticos.

- Establecer el funcionamiento de diferentes circuitos de polarización y comparar las características medidas de los transistores con las hojas de datos.

- Analizar un circuito en el que el transistor se comporta como interruptor, es decir, su punto de trabajo (Q) se mueve entre saturación y corte.

- Analizar un circuito en el que el transistor se comporta como un amplificador.

Una vez definido los objetivos el siguiente paso a dar es presentar la placa de circuito que se conectará en el laboratorio portátil de manera que permita cumplir con los objetivos de la clase.

Las consignas de trabajo se exponen como directivas en las que los estudiantes deben ir cumpliendo en principio con el objetivo de dominar la potencialidad de la placa y discriminar paso a paso las opciones de conexionado y cómo alimentar y medir el circuito para satisfacer la consigna. Luego en los siguientes ensayos el alumno debe, con directivas menos específicas, encontrar la forma de ir satisfaciendo las consignas.

De esta manera se fomenta el autoaprendizaje al tener la opción de variar componentes reales, de jugar con los parámetros de visualización por pantalla, etc.

Al final el alumno deberá presentar un informe de las experiencias convalidando los pasos del ensayo mediante la producción de los documentos necesarios que serán comunicados al docente mediantes herramientas TICs.

Es muy importante destacar que el software solo convalida que la directiva se haya cumplido, no la calidad de la información que se ha cargado, esto debe ser así para que la experimentación se acerque a la experiencia en un laboratorio real 
donde, como es lógico, existe la posibilidad de que al no tener afianzado los conocimientos se produzcan errores en el desarrollo de las mediciones y deba rehacer la práctica hasta subsanar los errores en las mediciones.

Por eso se vuelve muy importante en este laboratorio la potencialidad de poder cumplir con la etapa de evaluación de los resultados por parte del docente con la consiguiente realimentación, para que los alumnos puedan corregirse en un corto tiempo y con la realimentación lo más adecuada posible.

Finalmente en el diseño de la clase se debe dejar en claro el papel que deberán cumplir los docentes y los estudiantes.

El rol del docente es claramente la de un motivador por excelencia que estará convencido y por ello convencerá de que el aprendizaje debe centrarse en el sujeto que aprende. Para ello los profesores deben dar primacía a la enseñanza expositiva-deductiva, a través de un aprendizaje receptivo y activo, donde a partir de la comprensión del concepto general se llega a la del concepto específico. Debe saber cómo organizar los materiales para adquirir aprendizajes significativos, principalmente aquellos que se presentan primero en una tarea de aprendizaje con un nivel de abstracción e inclusión más amplia que la tarea misma, deben servir de puente entre el conocimiento previo y el material más diferenciado y detallado que se presentará posteriormente. Debe ser un guía, un facilitador de conceptos a través del análisis de sus atributos (característica que diferencia un concepto de otro), los ejemplos (las instancias o casos de un concepto), la definición (la regla que especifica los atributos esenciales de un concepto) y las relaciones jerárquicas (entre conceptos basados en sus atributos esenciales). Es decir que presentando los conceptos en forma ordenada se procura un proceso de búsqueda del concepto sin errores, llegando a contrastar lo esencial de lo que no lo es.

El alumno debe ser el protagonista casi exclusivo de su aprendizaje. En el aprendizaje experiencial esto no es un postulado abstracto sino todo lo contrario. Deben los los alumnos construir su propio aprendizaje desde las experiencias, es decir, construir, modificar, enriquecer y diversificar sus esquemas con la ayuda pedagógica que propicie condiciones para crear esa dinámica interna. La educación debe ser individualizada y centrada en el alumno; ambos principios derivados del constructivismo implican que es el alumno quien, ante la amplia diversidad de esquemas reinantes en el aula, selecciona sus propias experiencias de aprendizaje y el profesor es quien debe crear los medios para que cada uno lo pueda hacer basado en sus intereses y necesidades personales. Es allí que el uso de esta herramienta TICs tiene su mayor potencial. Es cada uno responsable directo de su aprendizaje, con la opción de hacer metodologías adicionales que propicien la interacción social, que también tiene un papel significativo en el aula, por cuanto los conocimientos lógicos y sociales se aprenden mejor dentro de un grupo de iguales.

\section{Conclusiones}

A partir de la integración del equipamiento descrito con la actividad académica dentro del Laboratorio de Electrónica, se logra cumplir las cuatro fases del modelo de aprendizaje experiencial.

Claro está que las prácticas realizadas en un entorno de laboratorio real 
constituyen actividades que permiten a los alumnos consolidar conocimientos a través de la experimentación con la realidad, la manipulación de equipos y datos, instrumentos y circuitos eléctricos, pero esto no siempre es así tal como puede deducirse de las indagaciones realizadas a alumnos que han cursado estos espacios curriculares entre 2015 y 2016.

Esta generación necesita de cambios constantes y que las TICs estén en el centro de la escena los motiva especialmente. Por eso es que se plantea esta nueva herramienta.

El grupo de investigación ha decidido antes de dar el toque inicial de lo que implica la construcción del equipo, priorizar el diseño de la clase de manera de tener claro los requerimientos que se le exigirá al sistema, para que no quede en el mero deseo de innovar en la enseñanza sino que realmente sea un instrumento innovador que sea el fiel reflejo de lo que las nuevas demandas exigen.

\section{Recomendaciones}

La corriente constructivista, actualmente en boga en el mundo de la enseñanza, habla de la complejidad del aprendizaje y cómo cada persona aprende de forma diferente (Martínez Delgado, 1999).

Hay innumerables formas de enseñar y aprender, cada uno debe buscar la metodología propia para su asignatura, se deben adaptar a las circunstancias y medios disponibles. No hay un método único e infalible que todos debamos descubrir y copiar. Tampoco se deben tomar los procedimientos comunes e incorporarlos sin adaptar a las propias convicciones y necesidades.

Parte de lo expuesto puede servir a otros profesores para diseñar sus propias asignaturas y metodologías.

Cualquier cambio es conveniente realizarlo de manera progresiva. Debe probarse nuevos métodos, valorarlos y corregirlos, hasta encontrar la manera de enseñar con la mayor eficacia. De cualquier forma la innovación siempre ayuda a mejorar.

Durante estos años se han ido introduciendo y probando distintas tareas, en ocasiones han servido para mejorar, otras veces para no caer en la rutina de hacer lo mismo todos los años y otras para adaptarse a las circunstancias de un determinado curso.

La mayor dificultad que se ha encontrado durante estos años es la falta de experiencia de los estudiantes en la partición. En muy pocas asignaturas se les anima a ser activos, a trabajar cada día, o exponer sus puntos de vista en clase. A medida que todos los profesores vayan incorporando técnicas de este estilo, los estudiantes estarán más acostumbrados a trabajar, participar y aprender haciendo, que a estudiar los últimos días para aprobar.

Otra dificultad común a numerosas asignaturas es la falta de motivación, el estudiar para tener un título no les seduce. Quieren aprender para ejercer una profesión y, por tanto, es muy importante acercarles a la realidad. La ingeniería no es un conjunto de teorías y fórmulas, es una actividad profesional creativa que requiere un contacto con las necesidades de la sociedad, por ello es fundamental adquirir las competencias necesarias durante su formación para poder desempeñarse con éxito. 


\section{Bibliografía}

González, M. L., Marchueta, J., Vilche, E. A. (s/a). “Modelo de aprendizaje experiencial de Kolb aplicado a laboratorios virtuales en Ingeniería en Electrónica”. UNITEC (Unidad de Investigación y Desarrollo para la Calidad de la Educación en Ingeniería con orientación al uso de TIC), Facultad de Ingeniería, U. N. L. P. Disponible en <http://www.unlp.edu.ar/uploads/docs/modelo_de_ aprendizaje_experiencial_de_kolb_aplicado_a_laboratorios_virtuales_en_ ingenieria_en_electronica_gonzalez_y_otros_.pdf >.

Kolb, D. (2010). "Learning styles and disciplinary differences". Disponible en $<$ http://learningfromexperience.com/media/2010/08/Learning-styles-anddisciplinary-difference.pdf $>$.

Kolb, R. y McIntyre (1974). "Modelo de David Kolb, aprendizaje basado en experiencias”. Disponible en <http://www.cca.org.mx/profesores/cursos/cep21/ modulo_2/modelo_kolb.htm>.

Martínez Delgado, A. (1999). “Constructivismo Radical, Marco Teórico de Investigación y Enseñanza de las Ciencias” en UAB, Enseñanza de las ciencias, 17 (3), 493-502. Disponible en <http://e-spacio.uned.es/fez/eserv/ taee:congreso-2006-1059/S2A02.pdf $>$. 